\title{
Electrocardiogram Differences in Reperfusion Phase of Kidney Transplantations From Living Donors
}

B. Dinç ${ }^{1}$, I. O. Aycan ${ }^{1}$, N. Cal Hadimioglu ${ }^{1}$, Z. Ertug ${ }^{1}$, A. Kisaoglu ${ }^{2}$, B. Aydinli²

${ }^{1}$ Akdeniz University Medical Faculty Department of Anesthesiology and Reanimation - Antalya (Turkey), ${ }^{2}$ Akdeniz University Medical Faculty Department of general surgery - Antalya (Turkey)

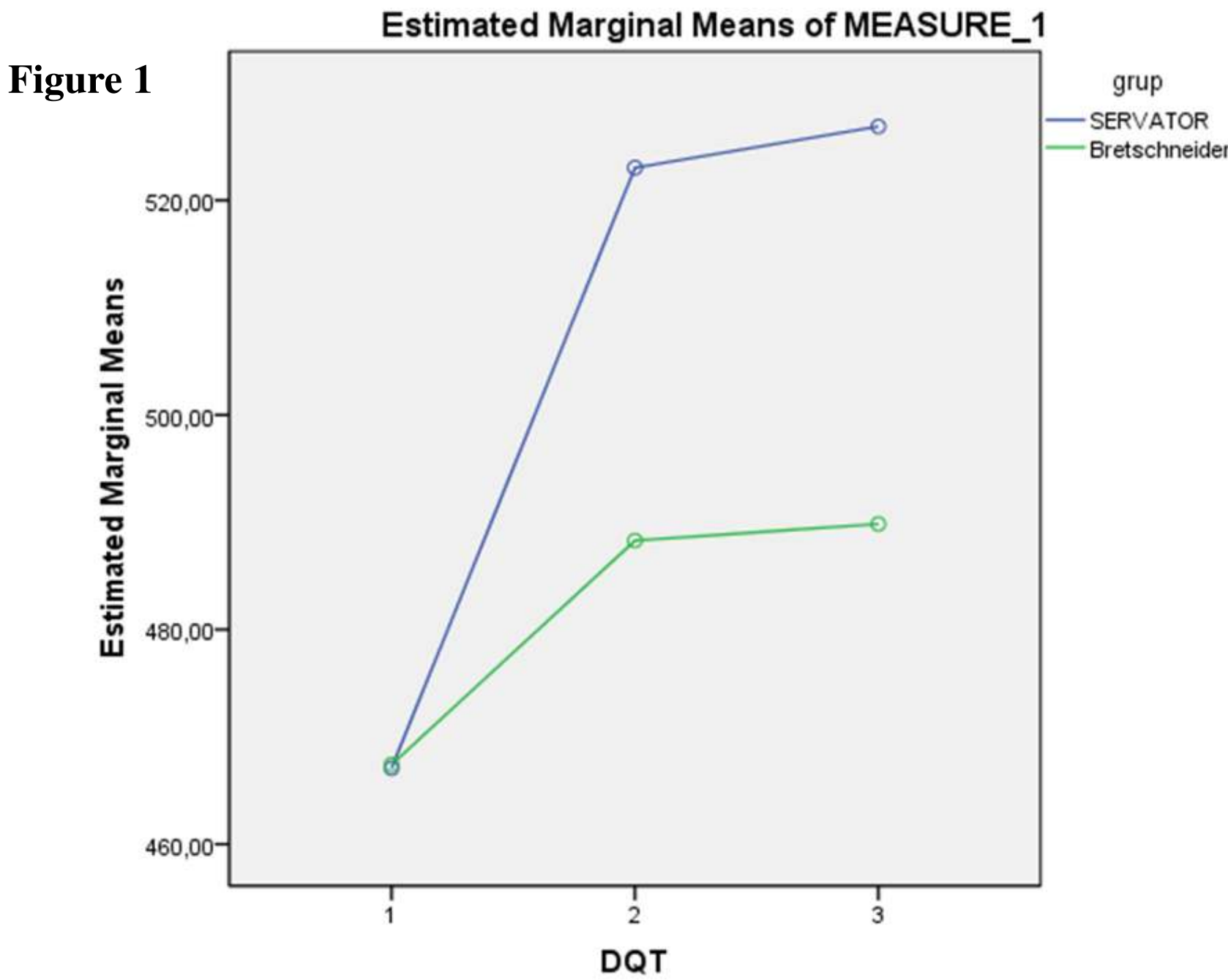

Background and Goal of Study:

Graft reperfusion, a phase in kidney transplantation, may show rapid changes in hemodynamic parameters. The purpose of this study is to be able to detect the hemodynamic and metabolic alterations between the two solutions with EKG variations that may occur following the kidney graft reperfusion.

\section{Materials and Methods:}

While Group $1(\mathrm{n}=40)$ uses university of wisconsin solution (Servator B SALF (S.A.L.F S.p.A., Cenate Sotto, Italy) solution), Group 2 $(n=40)$ uses Histidine-tryptophan-ketoglutarate solution (Bretschneider modificata (Galenica Senede, Siena, Italy) solution). Living kidney transplantation cases that do not have any cardiac complaints were included in the study. Demographic data such as age, sex, ASA as well as preoperative, perfusion and postoperative EKGs were taken at the same time with the blood gases. Hot and cold ischemia times of the kidney graft were recorded according to the amounts of graft wash solution used.

\section{Results and Discussion:}

No differences were observed in terms of demographic data, operation periods, hot and cold ischemia times, blood gas analyses and electrolytic changes. In Group 1, 34 patients were observed to have cardiac problems (Bradycardia: 19, Asystole: 8, Inotropic treatment: 7 patients). The incidence of bradycardia, asystole and inotrope adjustments between groups were shown at table 1 . No cardiac changes were observed in Group 2. Comparing Group 1 and Group 2, while preoperative corrected QT interval (cQT) was not found to be different $(\mathrm{p}=0,981)$, perfusion cQT $(\mathrm{p}=0,01)$ and postoperative cQT $(\mathrm{p}=0,014)$ were found to be significantly different. In Group 1, perfusion cQT $(\mathrm{p}=0,001)$ and postoperative $\mathrm{cQT}(\mathrm{p}=0,001)$ were observed to differ from preoperative cQT. There were no differences between perfusion $\mathrm{cQT}$ and postoperative $\mathrm{cQT}(\mathrm{p}=0,575)$. In Group 2, perfusion cQT $(\mathrm{p}=0,019)$ and postoperative cQT $(\mathrm{p}=0,026)$ were observed to differ from preoperative $\mathrm{cQT}$. There were no differences between perfusion $\mathrm{cQT}$ and postoperative cQT $(\mathrm{p}=0,825)$. (Table 2).

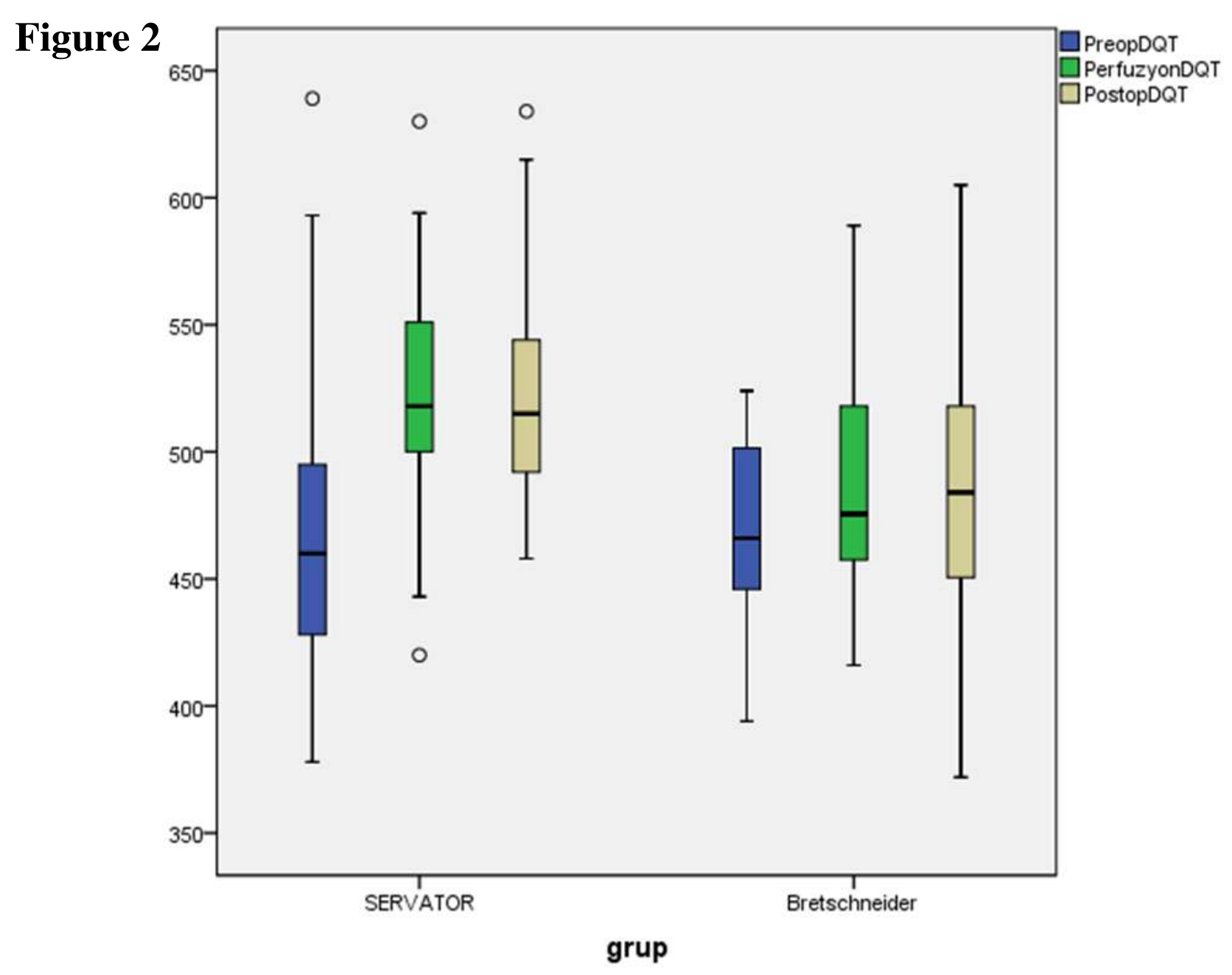

Table 1

\begin{tabular}{|c|c|c|c|c|c|c|c|}
\hline & & \multicolumn{2}{|c|}{ SERVATOR } & \multicolumn{2}{|c|}{ Bretschneider } & \multirow[b]{2}{*}{$p$} & \multirow[b]{2}{*}{ Test } \\
\hline & & $n$ & $\%$ & $n$ & $\%$ & & \\
\hline \multirow[t]{2}{*}{ BRADYCARDIA } & 0 & 20 & $51,3 \%$ & 40 & $100,0 \%$ & $<0,001$ & $\begin{array}{l}\text { Pearson Chi- } \\
\text { Square }\end{array}$ \\
\hline & 1 & 19 & $48,7 \%$ & 0 & $0,0 \%$ & & \\
\hline \multirow[t]{2}{*}{ ASYSTOLE } & 0 & 31 & $79,5 \%$ & 40 & $100,0 \%$ & 0,002 & $\begin{array}{l}\text { Fisher's Exact } \\
\text { Test }\end{array}$ \\
\hline & 1 & 8 & $20,5 \%$ & 0 & $0,0 \%$ & & \\
\hline \multirow[t]{2}{*}{$\begin{array}{l}\text { INOTROPIC } \\
\text { TREATMENT }\end{array}$} & 0 & 32 & $82,1 \%$ & 40 & $100,0 \%$ & 0,005 & $\begin{array}{l}\text { Fisher's Exact } \\
\text { Test }\end{array}$ \\
\hline & 1 & 7 & $17,9 \%$ & 0 & $0,0 \%$ & & \\
\hline
\end{tabular}

Table 2

\begin{tabular}{|c|c|c|c|c|c|}
\hline & \multicolumn{2}{|c|}{ SERVATOR } & \multicolumn{2}{|l|}{ Bretschneider } & \multirow[b]{2}{*}{ Mean Difference; $p$} \\
\hline & $n$ & Mean $\pm S d$ & $n$ & Mean $\pm S d$ & \\
\hline PreopDQT & 40 & $463,6 \pm 56,23$ & 40 & $471,43 \pm 44,44$ & $-0,337 ; 0,981$ \\
\hline PerfuzyonDQT & 38 & $519,97 \pm 55,2$ & 40 & $491,68 \pm 46,19$ & 34,$748 ; 0,01^{*}$ \\
\hline PostopDQT & 26 & $526,27 \pm 47,4$ & 24 & $489,83 \pm 53,38$ & 37,$047 ; 0,014^{*}$ \\
\hline 1-2 (Mean Difference; $p$ ) & \multicolumn{2}{|c|}{$-55,960 ;<0,001^{*}$} & $-20,875 ; 0,019^{*}$ & & \\
\hline 1-3 (Mean Difference; $p$ ) & \multicolumn{2}{|c|}{$-59,800 ;<0,001^{*}$} & $-22,417 ; 0,026^{*}$ & & \\
\hline \multirow[t]{2}{*}{ 2-3(Mean Difference; p) } & \multicolumn{2}{|c|}{$-3,840 ; 0,575$} & $-1,542 ; 0,825$ & & \\
\hline & $F$ & $p$ & Effect Size $\eta^{2}$ & Power & \\
\hline Main effect & 29,794 & $<0,001^{*}$ & 0,388 & 0,999 & \\
\hline Interaction effect & 6,183 & $0,005^{*}$ & 0,116 & 0,848 & \\
\hline
\end{tabular}

\section{Conclusion(s):}

Although hemodynamic and metabolic parameters are at normal levels during kidney transplantation, particularly in graft reperfusion stage, the kidney transplantation bears a risk in terms of disorders in ventricular repolarization period. Evaluation of QT interval can be an essential parameter in order to take required precautions and foresee complications. Whilst preoperative EKG of the cases provides warning, electrolyte levels must also be taken into consideration.

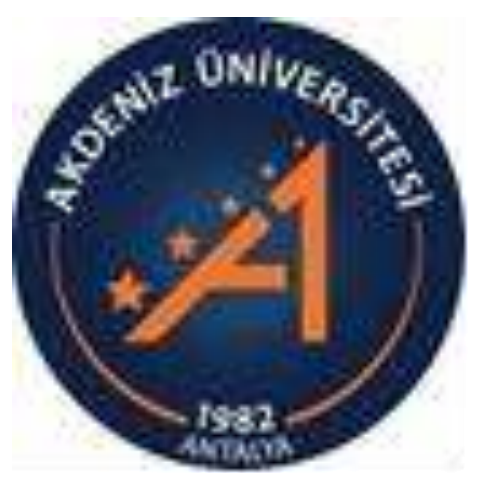

